\title{
TRACES AND SUBADDITIVE MEASURES ON PROJECTIONS IN JBW-ALGEBRAS AND VON NEUMANN ALGEBRAS
}

\author{
L. J. BUNCE AND J. HAMHALTER
}

(Communicated by Palle E. T. Jorgensen)

\begin{abstract}
Let $P(M)$ be the projection lattice of an arbitrary JBW-algebra or von Neumann algebra $M$. It is shown that the tracial states of $M$ correspond by extension precisely to the subadditive probability measures on $P(M)$. The analogous result for normal semifinite traces is also proved.
\end{abstract}

A positive measure on the projection lattice $P(M)$ of a JBW-algebra $M$ is a function

$\mu: P(M) \rightarrow[0, \infty]$ such that $\mu(e+f)=\mu(e)+\mu(f)$ whenever $e f=0$.

Such a measure is said to be a probability measure if $\mu(1)=1$. A positive measure is said to be normal if $\mu\left(e_{\alpha}\right) \rightarrow \mu(e)$ whenever $e_{\alpha} \nearrow e$ and semifinite if for each projection $e$ there is a net of projections $e_{\alpha} / e$ with $\mu\left(e_{\alpha}\right)<\infty$, for all $\alpha$.

A subadditive measure on $P(M)$ is a positive measure such that

$$
\mu(e \vee f) \leq \mu(e)+\mu(f), \quad \text { for all } e, f \in P(M) .
$$

By the generalisation of Gleason's Theorem $[3,4]$ each probability measure on $P(M)$ extends uniquely to a (linear) state on $M$ whenever $M$ has no Type $I_{2}$ direct summand. But this theorem is false for Type $I_{2} \mathrm{JBW}$-algebras.

The aim of this paper is to show that, for every JBW-algebra $M$, each subadditive probability measure on $P(M)$ extends uniquely to a tracial state on $M$. A corollary is that each subadditive normal semifinite measure on $P(M)$ extends to a normal semifinite trace on $M$.

In particular, this involves showing that, for subadditive measures, Gleason's Theorem is true for all JBW-algebras (including Type $I_{2} \mathrm{JBW}$-algebras).

We note that traces on JBW-algebras have been extensively studied in the literature $[1,2,5,7,9]$ and have particular significance for the criterion of types. Our results have the particular virtue of providing a purely lattice- and measure-theoretic characterisation of traces on JBW-algebras.

Since the selfadjoint part of a von Neumann algebra is a JBW-algebra, we note that our results hold for all von Neumann algebras as well as for all JBWalgebras.

Received by the editors April 12, 1993.

1991 Mathematics Subject Classification. Primary 46L05, 46L30, 46L70.

(c) 1994 American Mathematical Society $0002-9939 / 94 \$ 1.00+\$ .25$ per page 
The main reference for the theory of JBW-algebras is [6], to which the reader is referred for any unmentioned details. In order to facilitate the present discussion we recall that a JBW-algebra is a real Banach dual space and that a Jordan algebra $(M, o)$ satisfies

$$
\left\|x_{0} y\right\| \leq\|x\|\|y\| \text { and }\|x\|^{2} \leq\left\|x^{2}+y^{2}\right\| \text {. }
$$

A JW-algebra is a JBW-algebra that can be realized as a weakly closed Jordan algebra of selfadjoint operators on a Hilbert space. The Type $I_{2} \mathrm{JBW}$-algebras fall into this class and are $l^{\infty}$-sums of algebras of the form $L^{\infty}(\Omega, \mu, V)$, where $V$ is a spin factor and $\mu$ is a Radon measure on a locally compact Hausdorff space $\Omega$ [10].

A trace on a JBW-algebra $M$ is a mapping $\tau: M^{+} \rightarrow[0, \infty]$ for which

(i) $\tau(x+y)=\tau(x)+\tau(y)$, for all $x, y \in M^{+}$.

(ii) $\tau(\lambda x)=\lambda \tau(x)$, for all $x \in M^{+}$and $\lambda \geq 0$.

(iii) $\tau\left(U_{x}\left(y^{2}\right)\right)=\tau\left(U_{y}\left(x^{2}\right)\right)$, for all $x, y \in \bar{M}$.

The notation used in (iii) is explained as follows. For each $x$ in $M, U_{x}: M \rightarrow$ $M$ is the positive map defined by $U_{x}(y)=2 x_{0}\left(x_{0} y\right)-x_{0}^{2} y$. (When $M$ is a JW-algebra with product $x_{0} y=\frac{1}{2}(x y+y x)$, this reduces to $U_{x}(y)=x y x$.) If $\tau(1)=1$, then $\tau$ extends uniquely to a tracial state of $M$.

Let $e$ and $f$ be projections in a JBW-algebra $M$, and let $\tau$ be a trace on $M$. By [5, Lemma 2.3] together with [11, Corollary 8] there is a symmetry $s$ in $M$ with

$$
U_{s}(e \vee f-f)=e-e \wedge f
$$

from which it follows easily that $\tau(e \vee f) \leq \tau(e)+\tau(f)$. Hence, every trace on $M$ restricts to a subadditive measure on $P(M)$.

Theorem. Let $M$ be a JBW-algebra, and let $\mu$ be a subadditive probability measure on $P(M)$. Then $\mu$ extends uniquely to a tracial state on $M$.

Proof. Being a probability measure, $\mu$ extends uniquely to a quasi-linear functional $\bar{\mu}$ on $M[3,4]$. That is, $\bar{\mu}$ is linear (and continuous) on all abelian subalgebras of $M$.

(i) Let $M$ be a Type $I_{2}$ JBW-algebra. Let $x, y \in M$. We have to show that

(a) $\bar{\mu}(x+y)=\bar{\mu}(x)+\bar{\mu}(y)$ and

(b) $\bar{\mu}\left(x y^{2} x\right)=\bar{\mu}\left(y x^{2} y\right)$.

Since the JBW-algebra, $M(x, y)$, of $M$ generated by $x$ and $y$ is a direct sum of an abelian algebra and a Type $I_{2}$ algebra, no harm can can come from supposing that $M=M(x, y)$. In which case, let $\pi$ be a factor representation of $M$. Then $\pi: M \rightarrow V$ where $V$ is a spin factor and $\pi(M)=V$. So

$$
\pi(x)=a 1+\beta e, \quad \pi(y)=\lambda 1+\mu f
$$

where $e, f$ are minimal projections in $V$ and $\alpha, \beta, \lambda, \mu \in \mathbb{R}$. But (simple calculation) the Jordan subalgebra of $V$ generated by $e$ and $f$ is linearly generated by $e, f$ and $e f+f e$, from which it follows that $V=M_{2}(\mathbb{R})_{s a}$. By [10, Theorem 2], for example, we may therefore suppose that

$$
M=C(X) \otimes M_{2}(\mathbb{R})_{s a},
$$

where $C(X)$ is the algebra of all continuous real-valued functions on a compact hyperstonean space $X$. 
We note that $\mu(e)=\mu(f)$ whenever $e, f \in P(M)$ satisfy

$$
e \vee(1-f)=1=(1-e) \vee f \text {. }
$$

Indeed, because $\mu$ is subadditive, the first equation of (1) implies that

$$
1 \leq \mu(e)+\mu(1-f)=\mu(e)+1-\mu(f), \quad \text { so that } \mu(f) \leq \mu(e) .
$$

Let $e, f \in P(M)$ with $\|e-f\|<1$. Then $e \wedge(1-f)=0$, because

$$
e \wedge(1-f)=(e-f)(e \wedge(1-f)) \text {. }
$$

Hence, $(1-e) \vee f=1$. Similarly, $e \vee(1-f)=1$. So $\mu(e)=\mu(f)$, by (1). In particular, $\mu$ is uniformly continuous on $P(M)$.

Suppose now that $e$ is any nontrivial projection $(\neq 0,1)$ in $N=1 \otimes$ $M_{2}(\mathbb{R})_{s a}$. Choose a projection $f \neq 1$ in $N$ such that $e f \neq 0$. Then applying (1) to each of the pairs $e, f$ and $1-e, f$ in turn, we see that

$$
\mu(e)=\mu(f)=\mu(1-e), \quad \text { so that } \mu(e)=\frac{1}{2} .
$$

By the unicity of quasi-state extensions this means that $\bar{\mu}$ can be none other than the (linear) trace on $N$. Since for any projection $z$ of $C(X)$ there is a compact hyperstonean subspace $Y$ of $X$ with $z C(X)=C(Y)$ and $z M=$ $C(Y) \otimes M_{2}(\mathbb{R})_{s a}$, it follows that $\bar{\mu}$ is a (linear) trace on $z \otimes M_{2}(\mathbb{R})_{s a}$. Thus, letting $A$ be the Jordan subalgebra of $M$ generated by all finite sums of the form

$$
\sum_{1}^{n} z_{i} \otimes M_{2}(\mathbb{R})_{s a} \quad \text { where the } z_{i} \in P(C(X)) \text { and } \sum_{1}^{n} z_{i}=1(n \in \mathbb{N}),
$$

it is immediate that $\bar{\mu}$ is linear on $A$ and that $\bar{\mu}\left(x y^{2} x\right)-\bar{\mu}\left(y x^{2} y\right)$, for all $x, y \in A$. But $A$ is uniformly dense in $M$ and also the set of projections of $A$ is uniformly dense in $P(M)$ (cf. [8, Lemma 8.3]). It follows directly from this, together with the above observed continuity of $\bar{\mu}$ on $P(M)$, that $\bar{\mu}$ is linear on $M$ and that $\bar{\mu}\left(x y^{2} x\right)=\bar{\mu}\left(y x^{2} y\right)$, for all $z, y \in M$. In other words, $\bar{\mu}$ is a tracial state of $M$.

(ii) Now let $M$ be any JBW-algebra. By (i) and [3, 4] we may suppose that $\bar{\mu}$ is linear on $M$. Let $e, f \in P(M)$. Since the JBW-subalgebra of $M$ generated by $e$ and $f$ is of the form Type $I_{1} \oplus$ Type $I_{2}$, a further application of (i), above, together with part (iii) of [9, Theorem], implies that $\bar{\mu}\left(e_{0} f\right) \geq 0$ So, by spectral theory, $\bar{\mu}\left(x_{0} y\right) \geq 0$ for all $x, y \in M_{+}$, and the above-quoted result of [9] now implies that $\bar{\mu}$ is a tracial state of $M$.

Corollary. Let $\mu$ be a subadditive normal semifinite measure on the projections $P(M)$ of a JBW-algebra $M$. Then $\mu$ extends uniquely to a normal semifinite trace on $M$.

Proof. The subadditivity of $\mu$ means that $S=\{e \in P(M): \mu(e)<\infty\}$ is a sublattice of $P(M)$. In particular, $S$ is an increasing net which, by the semifiniteness of $\mu$, converges strongly to 1 . By the theorem and the fact that $\mu$ is normal, for each $e \in S$ the restriction of $\mu$ to $P\left(U_{e}(M)\right)$ extends uniquely to a finite normal tracial linear map $\mu_{e}$ on $U_{e}(M)$. The unicity of these extensions induces a linear mapping

$$
\mu_{0}: \bigcup_{e \in S} U_{e}(M) \rightarrow \mathbb{R}, \quad \text { with } \mu_{0}(x)=\mu_{e}(x) \text { if } x \in U_{e}(M),
$$


extending $\mu$. Given $e, f \in S$, with $e \leq f$ and $x \in M_{+}$, we have, for $a=U_{f}(x)^{1 / 2}$,

$$
\mu_{0}\left(U_{e}(x)\right)=\mu_{0}\left(U_{e}\left(a^{2}\right)\right)=\mu_{0}\left(U_{a}(e)\right) \leq \mu_{0}\left(a^{2}\right)=\mu_{0}\left(U_{f}(x)\right)
$$

Consequently, denoting $S$ by $\left\{e_{\alpha}\right\}$ we may define a mapping

$$
\bar{\mu}: M_{+} \rightarrow[0, \infty] \text { by } \bar{\mu}(x)=\lim \mu_{0}\left(U_{e_{\alpha}}(x)\right)
$$

which extends $\mu_{0}$ on $M_{+}$and satisfies, for $x, y \in M_{+}$and $\lambda \geq 0$,

$$
\bar{\mu}(x+y)=\bar{\mu}(x)+\bar{\mu}(y) \text { and } \bar{\mu}(\lambda x)=\lambda \bar{\mu}(x) .
$$

Now, with $p_{\alpha \beta}=e_{\alpha} \vee e_{\beta}, \bar{\mu}$ is a finite normal trace on $U_{p_{\alpha \beta}}(M)$, so that for $x, y \in M$ we have that

$$
\bar{\mu}\left(U_{e_{\alpha}} U_{x} U_{y}\left(e_{\beta}\right)\right)=\bar{\mu}\left(U_{e_{\beta}} U_{y} U_{x}\left(e_{\alpha}\right)\right) .
$$

Fixing $\beta$ and taking limits, this gives

$$
\bar{\mu}\left(U_{x} U_{y}\left(e_{\beta}\right)\right)=\bar{\mu}\left(U_{e_{\beta}} U_{y}\left(x^{2}\right)\right) \rightarrow \bar{\mu}\left(U_{y}\left(x^{2}\right)\right) .
$$

To see that $\bar{\mu}$ must be normal, let $a_{i} \uparrow a$ in $M_{+}$. Then, respectively, $\bar{\mu}\left(U_{e_{\alpha}}\left(a_{i}\right)\right)$ and $\bar{\mu}\left(U_{e_{\alpha}}(a)\right)$ increase to $\bar{\mu}\left(a_{i}\right)$ and $\bar{\mu}(a)$ with respect to $\alpha$ while $\bar{\mu}\left(U_{e_{\alpha}}\left(a_{i}\right)\right)$ increases to $\bar{\mu}\left(U_{e_{\alpha}}(a)\right)$ with respect to $i$. It follows that $\bar{\mu}\left(a_{i}\right) \rightarrow \bar{\mu}(a)$. So $\bar{\mu}$ is normal. The latter applied to (3) implies that $\bar{\mu}\left(U_{x}\left(y^{2}\right)\right)=\bar{\mu}\left(U_{y}\left(x^{2}\right)\right)$, for all $x, y$ in $M$, which together with (2) shows $\bar{\mu}$ to be a normal trace on $M$. The semifiniteness of $\bar{\mu}$ is an immediate consequence of $U_{a^{1 / 2}}\left(e^{\alpha}\right) \leq a$ and $\bar{\mu}\left(U_{a^{1 / 2}}\left(e_{\alpha}\right)\right)=\bar{\mu}\left(U_{e_{\alpha}}(a)\right)<\infty$, for all $a$. This completes the proof.

\section{REFERENCES}

1. S. A. Ajupov, Extension of traces and type criterions for Jordan algebras of self-adjoint operators, Math. Z. 181 (1982), 253-268.

2. S. A. Ajupov and R. Z. Abdullev, The Radon Nikodym Theorem for weights on JBWalgebras, Math. Z. 188 (1985), 475-484.

3. L. J. Bunce and J. D. M. Wright, Quantum measures and states on Jordan algebras, Comm. Math. Phys. 98 (1985), 187-202.

4. Continuity and linear extensions of quantum measures on Jordan algebras, Math. Scand. 64 (1989), 300-306.

5. U. Haagerup and H. Hanche-Olsen, Tomita-Takesaki Theory for Jordan algebras, J. Operator Theory 11 (1984), 343-364.

6. H. Hanche-Olsen and E. Størmer, Jordan operator algebras, Pitman, New York, 1984.

7. B. Iochum, Cônes autopolaires et algèbres dde Jordan, Lecture Notes in Math., vol. 1049, Springer-Verlag, New York, 1984.

8. S. Maeda, Probability measures on projections in von Neumann algebras, Rev. Math. Phys. 1 (1990), 235-290.

9. G. K. Pedersen and E. Størmer, Traces on Jordan algebras, Canad. J. Math. 34 (1982), 370-373.

10. P. J. Stacey, Type $I_{2}$ JBW-algebras, Quart. J. Math. Oxford 33 (1982), 115-127.

11. D. Topping, Jordan Algebras of self-adjoint operators, Mem. Amer. Math. Soc., vol. 53, Amer. Math. Soc., Providence, RI, 1965.

Department of Mathematics, Reading University, PO Box 220, Reading RG6 2AX, ENGLAND

Department of Mathematics, Technical University of Prague, Technicka 2, 16627 Prague 6, Czechoslovakia 